\title{
Abnormal white matter integrity in Chinese young adults with first-episode medication-free anxious depression: a possible neurological biomarker of subtype major depressive disorder
}

This article was published in the following Dove Press journal:

Neuropsychiatric Disease and Treatment

\author{
Weiping Xia ${ }^{1,2}$ \\ Rubai Zhou' \\ Guoqing Zhao' \\ Fan Wang' \\ Ruizhi Mao' \\ Daihui Peng' \\ Tao Yang' \\ Zuowei Wang ${ }^{1,3}$ \\ Jun Chen' \\ Yiru Fang ${ }^{1,4,5}$ \\ 'Division of Mood Disorders, \\ Shanghai Mental Health Center, \\ School of Medicine, Shanghai Jiao \\ Tong University, Shanghai, People's \\ Republic of China; ${ }^{2}$ Department of \\ Medical Psychology, Xinhua Hospital, \\ School of Medicine, Shanghai Jiao \\ Tong University, Shanghai, People's \\ Republic of China; ${ }^{3}$ Mood Disorder \\ Department, Hongkou District Mental \\ Health Center of Shanghai, Shanghai, \\ People's Republic of China; ${ }^{4}$ State Key \\ Laboratory of Neuroscience, Shanghai \\ Institutes for Biological Sciences, CAS, \\ Shanghai, People's Republic of China; \\ ${ }^{5}$ Shanghai Key Laboratory of Psychotic \\ Disorders, Shanghai, People's Republic \\ of China
}

Correspondence: Jun Chen; Yiru Fang Division of Mood Disorders, Shanghai Mental Health Center, School of Medicine, Shanghai Jiao Tong University, 600 South Wan Ping Road, Shanghai 200030, People's Republic of China Email doctorcj2010@gmail.com; yirufang@aliyun.com
Background: Almost half of patients with major depressive disorder (MDD) also have clinically meaningful levels of anxiety. Anxious depression is a distinct clinical subtype of MDD, which has poor response to pharmacotherapy; however, the neural mechanisms behind are largely unknown. In the present study, we explored the white matter (WM) integrity traits of anxious depression in first-episode and medication-free (medication-naïve and medication washout) Chinese young adult patients by detecting differences in diffusion tensor imaging (DTI) with the tract-based spatial statistics (TBSS) method.

Subjects and methods: DTI was obtained from 39 first-episode, medication-free anxious depressive patients, 45 nonanxious depressive patients, and 50 demographically similar healthy controls. All subjects underwent clinical assessments. TBSS was carried out to investigate the difference in WM integrity among three groups within DTI parameter maps. WM integrity was measured using fractional anisotropy (FA), mean diffusivity, axial diffusivity, and radial diffusivity (RD). The correlations between WM integrity and clinical features were also computed.

Results: When compared with nonanxious patients, lower FA values in anxious depressive patients were found in multiple regions of the brain, mainly involving left uncinate fasciculus (UF), superior longitudinal fasciculus (SLF), and forceps major and minor. Higher RD in forceps major and minor and SLF were also detected. The decreased FA values and increased RD values correlated with both anxiety level and depression level in the pooled depressive group.

Conclusion: The anxious depressive patients had more abnormalities in WM integrity at the early phase than the nonanxious group. Alternations in WM integrity in fiber pathways, including SLF, UF, and forceps major and minor, may play a critical role in the neuropathology of anxious depression and might help to identify anxious MDD from nonanxious MDD. Further study with larger sample size, larger age range, and longitudinal design is needed to confer a robust inference to better understand the dynamic neurological change and neuropathology of WM integrity in anxious MDD.

Keywords: diffusion tensor imaging, anxious depression, tract-based spatial statistics, microstructure of whiter matter

\section{Introduction}

Major depressive disorder (MDD) is a heterogeneous condition rather than a unitary disease. Various subtypes with different clinical features have been proposed. ${ }^{1,2}$ Within all the clinical subtypes, anxious depression is common and clinically relevant. It has 
been estimated that up to $40 \%-50 \%$ of MDD patients of mixed-age suffered from anxiety. ${ }^{3-5}$ Clinically, anxious depressive patients have a unique course of illness characterized by more severe depressive symptoms and greater severity of functional impairment. ${ }^{6-9}$

Although evidence exists that anxious depression is a clinically distinct diagnosis, the neurophysiological mechanism associated is largely unknown. Given the dysfunction of white matter (WM) fiber pathways may be one of the most important pathogenesis involved in anxious depressive patients. Diffusion tensor imaging (DTI), one of the most widely used noninvasive methods to detect the WM integrity in vivo, might help us better to explore the underlying biology of this "disconnection syndrome" and improve the consensus in diagnosis and treatment. ${ }^{10-13}$

As a highly sensitive but nonspecific summary measure of WM microstructure derived from DTI, fractional anisotropy (FA) is a measure of anisotropic water diffusion that reflects the degree of directionality of cellular structures within the fiber tracts. ${ }^{14-16}$ Mean diffusivity (MD), radial diffusivity (RD), and axial diffusivity (AD) are additional measures that may reflect edema, myelination, and axonal injury, respectively. ${ }^{17,18}$

Within the main analysis techniques, region of interest (ROI) analysis and voxel-based morphometry have obvious shortcomings, while tract-based spatial statistics (TBSS), as an automated voxel-based method, could make the DTI data analysis more sensitive, objective, and interpretable. ${ }^{19}$

Previous studies using whole-brain analysis suggested that there are widespread WM abnormalities in MDD and its subtypes, including the frontal gyrus, ${ }^{20-22}$ right thalamus, ${ }^{23,24}$ uncinate fasciculus (UF), ${ }^{25}$ corpus callosum (CC), ${ }^{26,27}$ right parietal lobe, ${ }^{21,22}$ superior longitudinal fasciculus (SLF), ${ }^{28-30}$ and cerebellum. . $^{21,23,24}$

However, these results are inconsistent, with studies finding alternations ${ }^{25}$ and also studies finding no differences, ${ }^{31}$ probably owing to the heterogeneity of subjects (such as differences in age, medication, illness duration, and the clarification of clinical subtypes) and methodological differences in the previous studies; it is unclear about which neuroimaging changes observed in the literatures are specifically related to subtype MDD and which are related to MDD in general.

Therefore, information such as the age of the sample, medication details, and duration of illness requires qualification and clarification.

To date, the majority of DTI studies in MDD have focused on late-life or mixed age patients. ${ }^{32-36}$ Younger adulthood was a risk period for the emergence of depression and could be more severely impacted. ${ }^{34}$ Moreover, myelination and WM volume were reported to change with age. Decreased FA values and increased MD and RD values are commonly detected in normal aging. ${ }^{37,38}$ Besides, the onset age of anxious symptoms and anxiety disorders is ranging from adolescence to young adulthood. As we focus on anxious subtype of depression, it is important to focus on the younger adults. ${ }^{39}$

The impact of antidepressants on WM is not well characterized. Owing to inclusion of medicated patients in the studies, it is difficult to conclude whether these reported alterations are associated with MDD or confounded by medication effects. ${ }^{11,40-42}$

Since chronic or long duration illness may lead to more severe neuroimaging change, ${ }^{43}$ progressive WM loss could happen during the duration of illness. ${ }^{44}$ First-episode patients with short duration of illness may help us to better understand what happens at the early stage of illness.

In the present study, focusing on the anxious subtype of MDD, we measured WM integrity in first-episode, medication-free young adults with anxious and nonanxious depressions as well as matched healthy controls using TBSS methods. We chose to use the dimensional way to assess anxious depression, which is more clinically feasible. ${ }^{38,45}$

We hypothesized that, compared to healthy controls and the nonanxious depressive ones, anxious depression would show lower FA (together with alternations in other DTI measures) in the WM fiber bundle, which means more severe impairment in WM, causing dysfunction in the fiber pathways related to emotional and cognitive regulations, and that this change may be correlated with clinical features.

\section{Subjects and methods Subjects}

The study was approved by the Research Ethics Committee of Mental Health Center, School of Medicine, Shanghai Jiao Tong University. All subjects included in our study were provided written informed consent according to the Declaration of Helsinki. All subjects were Mandarin-speaking Han Chinese, aged between 20 and 40 years, right handed (determined by the Edinburgh Inventory of handedness), and with an education $>9$ years. The clinical trial number for this study is NCT03219008.

Patients were recruited at the out-patient clinic of Shanghai Mental Health Center (SMHC) from 2013 to 2016, who met Diagnostic and Statistical Manual of Mental Disorders, 4th edition, text revision ( $D S M$-IV-TR) major depressive disorder 
criterion. All were first-episode, medication-free depressive patients who were enrolled in our study within 2 days after the definite diagnosis.

Healthy controls were recruited from the community via poster advertisements. All matched with patients in age, gender, and education level. All passed our screening and had no history of psychosis or debilitating general medical disorders.

\section{Clinical interview}

Current MDD attack was ascertained by the use of a structured clinical interview (Mini International Neuropsychiatric Interview [MINI]) according to the DSM-IV-TR criteria to exclude subjects with other major current and past psychiatric Axis I comorbidity and Axis II comorbidity (personality disorders or mental retardation), which may influence the current mental status of the patients. Other exclusion criteria involved any psychotropic drug used within 4 weeks, alcohol or substance abuse/dependence within 6 months, contradiction of MRI (eg, metallic implants), medical conditions that would contribute to psychiatric symptoms, history of head trauma, a history of neurological illness, the item 3 score of Hamilton Rating Scale for Depression (HAMD)-17 $\geq 3$ (risk of suicide), and electroconvulsive therapy within 6 months before the present investigation. Exclusion criteria were the same for the healthy controls as in the patient groups.

All patients eligible for our study were given clinical assessment in which the demographic information was collected and clinical traits were assessed with the HAMD-17 and the Hamilton Rating Scale for Anxiety (HAMA).

\section{Anxious and nonanxious depressions}

Based on their scores on the HAMD at index assessment, subjects were divided into anxious and nonanxious groups. Anxious depression was defined as MDD with high levels of anxiety symptoms, as reflected in a HAMD-17 anxiety/ somatization factor score of $\geq 7$, which was used in our previous study ${ }^{45}$ and other studies. ${ }^{5,46,47}$ The anxiety/somatization factor of the HAMD-17 includes the following six items: psychic anxiety, somatic anxiety, gastrointestinal somatic symptoms, general somatic symptoms, hypochondriasis, and insight. ${ }^{48}$

\section{Image acquisition}

Subjects were scanned after recruiting in this research and finishing the clinical assessment. Imaging data were collected on a 3.0T Siemens Verio MRI scanner (Erlangen, Germany) at SMHC. A standard head coil with foam padding was used to restrict head motions. DTI was performed using a spin-echo single shot echo-planar pulse sequence (EPI) with a total of 20 different diffusion directions. Other parameters include repetition time (TR) $10,200 \mathrm{~ms}$, echo time (TE) $90 \mathrm{~ms}$, matrix $128 \times 128$, field of view (FOV) $256 \times 256 \mathrm{~mm}$, the number of excitations (NEX) 1 , and slice thickness $2 \mathrm{~mm}$. Images were inspected for movement artifacts and the homogeneity of image intensity immediately after scans and repeated if necessary.

\section{Data analysis}

Image processing

Diffusion-weighted images were preprocessed with functional MR imaging of the brain (FMRIB) Software Library (FSL) (http://www.fmrib.ox.au.uk/fsl). Following the TBSS guideline, ${ }^{19}$ we normalized individual FA volumes of the three groups to the Montreal Neurological Institute (MNI) space via affine registration. A mean FA image was created by averaging all the registered FA images, and from the mean FA image, the FA skeleton which represents the center of all WM tracts was created. Then, the FA threshold $(>0.2)$ was set on the skeleton to exclude the peripheral brain areas, including WM voxels only. All the registered FA images were further projected onto the skeleton, resulting in a $4 \mathrm{D}$ file of all skeletonized images from the individual subject. Then, we generated the AD, RD, and MD data by applying the FA nonlinear registration to the individual parametric maps and projecting them onto the skeleton. All DTI data were analyzed with protocols provided by TBSS. ${ }^{49}$

\section{Statistical analysis}

For the demographic and clinical features, one-way analysis of variance (ANOVA) and two-sample $t$-tests or chi-square test were performed to reveal group differences among the anxious depressive patients, nonanxious depressive patients, and healthy controls by SPSS Version 19.0.

ANOVA was performed to explore the FA, MD, RD, and $\mathrm{AD}$ differences among the three groups using the statistical analysis panel randomize v2.1 implemented in the FSL4.1.

Nonparametric permutation-based tests with 5,000 permutations and threshold-free cluster enhancement were used in the final statistical model. The findings were considered significant at a height threshold of $P<0.05$ (family-wise error corrected) adjusted for multiple comparisons by using the null distribution of the maximal voxel-wise test statistic, with age, gender, and education level as covariates. The Johns Hopkins University White-Matter Atlas was used to localize WM tracts with significant group differences. ${ }^{19}$ 
Post hoc analysis was performed if there were group differences in the DTI measures. Two sample $t$-tests were performed then, separately for every two groups, with special interest to the comparison between anxious depressive patients and nonanxious depressive patients. Furthermore, Cohen's $d$ was used to investigate the effect sizes.

Associations of WM alterations with clinical symptoms were further explored. Since there is conceptual and psychometric overlap, the HAMD total scores and HAMA total scores are highly correlated $(r=0.80, P<0.001),{ }^{50}$ we only ran the correlation between DTI measures and HAMA total scores, and the correlation between DTI measures and the HAMD-17 total scores was not run separately. Instead, we ran the correlation between DTI measures and the adjusted HAMD-17 scores, with anxiety/somatization factor (which we used to define the anxious or nonanxious subtype $\mathrm{MDD}^{45}$ ) excluded.

To investigate the relationship between the WM alterations with significant group differences and the HAMA total scores and adjusted HMAD-17 scores, Pearson correlation analyses were performed on the pooled depression patients, including anxious depressive patients and nonanxious depressive patients.

\section{Results}

\section{Demographic and clinical features}

Details on the demographic and clinical data are shown in Table 1.

As expected, there was no statistical significance observed for age, gender ratio, or years of education among the three groups. Illness durations were not significantly different between patient groups. Compared with the nonanxious patients, HAMD-17 and HAMA scores in the anxious group were significantly higher. Anxious subtype patients were more severely depressed even after adjustment for the severity of depression.

\section{Diffusion parameters among groups}

Group differences in diffusivity indices are shown in Table 1. There are significant differences in the FA and RD values, but no significant differences were found in the AD or MD values.

Figure 1 shows differences in FA and RD values between the two patient groups. Decreased FA was distributed over brain regions including forceps major and minor, left SLF, and left UF. RD values were significantly increased in brain regions including left SLF, left UF, forceps major, and forceps minor.

Table 2 shows the results that compared with nonanxious depressive patients, the anxious depressive patients displayed decreased FA in cluster-1, -2 , and -3 . As in the RD maps, the nonanxious depressive patients had significantly decreased $\mathrm{RD}$ values in cluster-1. The involving WM fiber tracts included left UF, left SLF, and bilateral forceps major and minor.

No increases in FA values or decreased RD values were detected in patients compared with healthy controls. We also did not find any brain cluster with higher FA or lower RD in anxious MDD compared with nonanxious MDD.

\section{Symptoms severity and DTI correlations}

Mean FA and RD values were extracted from certain brain clusters with significant group differences in FA parameter maps. As shown in Figure 2, in the pooled depression group, the HAMA total scores correlated negatively with both

Table I Demographics, clinical features, and DTI indices

\begin{tabular}{|c|c|c|c|c|c|}
\hline Measure (mean \pm SD) & $\begin{array}{l}\text { Anxious } \\
\text { depression } \\
(n=39)\end{array}$ & $\begin{array}{l}\text { Nonanxious } \\
\text { depression } \\
(n=45)\end{array}$ & $\begin{array}{l}\text { Healthy } \\
\text { controls } \\
(n=50)\end{array}$ & Value & $P$-value \\
\hline Age (years) & $26.35 \pm 6.31$ & $27.27 \pm 5.88$ & $27.55 \pm 5.50$ & 0.634 & $0.852^{*}$ \\
\hline Education (years) & $15.84 \pm 3.12$ & $|5.2| \pm 3.29$ & $15.52 \pm 3.23$ & 0.413 & $0.664 *$ \\
\hline Gender (male/female) & $16 / 23$ & $18 / 27$ & $21 / 29$ & 3.347 & $0.181 * *$ \\
\hline Duration of illness (months) & $12.43 \pm 8.19$ & $11.43 \pm 8.36$ & - & 2.517 & $0.121 * * *$ \\
\hline HAMD & $23.44 \pm 5.47$ & $18.63 \pm 4.36$ & - & 10.537 & $0.000 * * *$ \\
\hline Adjusted HAMD ${ }^{\mathrm{a}}$ & $|5.3| \pm 5.12$ & $13.50 \pm 4.05$ & - & 3.131 & $0.028 * * *$ \\
\hline HAMA & $21.80 \pm 6.32$ & $12.45 \pm 5.04$ & - & 13.48 & $0.000 * * *$ \\
\hline $\mathrm{FA}\left(\mathrm{mm}^{2} / \mathrm{s}\right)$ & $0.528 \pm 0.013$ & $0.659 \pm 0.017$ & $0.755 \pm 0.019$ & 5.675 & $0.004^{*}$ \\
\hline $\mathrm{MD}\left(10^{-4} \mathrm{~mm}^{2} / \mathrm{s}\right)$ & $8.581 \pm 0.217$ & $8.743 \pm 0.231$ & $8.622 \pm 0.224$ & 1.012 & 0.365 \\
\hline $\mathrm{RD}\left(10^{-4} \mathrm{~mm}^{2} / \mathrm{s}\right)$ & $6.232 \pm 0.233$ & $5.75 I \pm 0.265$ & $5.244 \pm 0.262$ & 5.041 & $0.010 *$ \\
\hline $\mathrm{AD}\left(10^{-3} \mathrm{~mm}^{2} / \mathrm{s}\right)$ & $1.121 \pm 0.022$ & I. $123 \pm 0.020$ & $1.128 \pm 0.023$ & 0.375 & 0.708 \\
\hline
\end{tabular}

Notes: *P-values for one-way ANOVA. **P-values for chi-square test. ***P-values for two-sample $t$-tests. ${ }^{2}$ Anxiety/somatization factor excluded.

Abbreviations: AD, axial diffusivity; ANOVA, analysis of variance; DTI, Diffusion Tensor Imaging; FA, fractional anisotropy; HAMA, Hamilton Rating Scale for Anxiety; HAMD, Hamilton Rating Scale for Depression; MD, mean diffusivity; RD, radial diffusivity. 

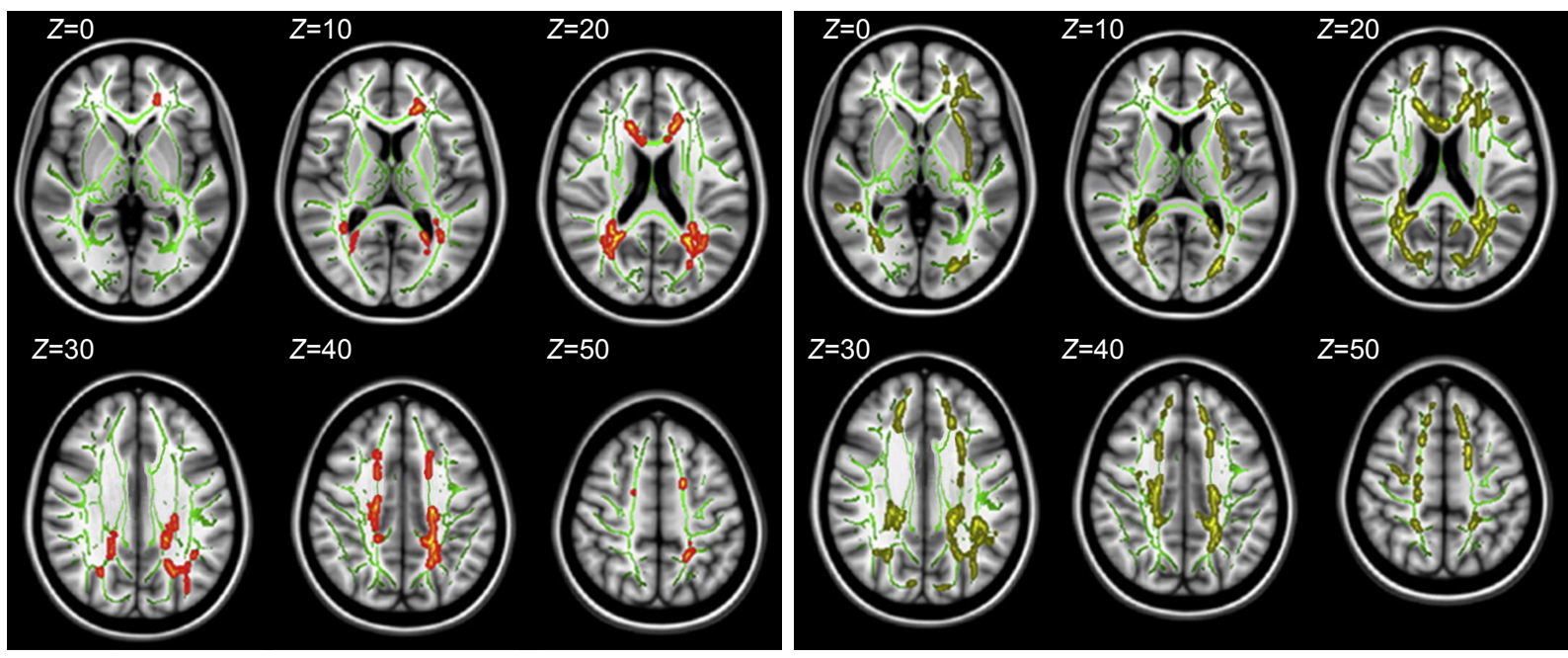

Figure I TBSS analysis of FA and RD volumes revealed lower FA values (in red-yellow) and higher RD values (in yellow) in anxious MDD group when compared with the nonanxious group.

Notes: Results are overlaid on axial slices from the MNI standard brain at a permutation-based threshold of $P<0.05$ (FWE corrected). The background images are TI-weighted MNI template brains denoted with MNI coordinates. No regions of increased FA or decreased RD were found in the groups.

Abbreviations: FA, fractional anisotropy; FWE, family-wise error; MDD, major depressive disorder; MNI, Montreal Neurological Institute; RD, radial diffusivity; TBSS, tract-based spatial statistics.

the mean FA values in cluster-1 and -3 and also correlated positively with RD values in cluster-1. And the adjusted HAMD-17 scores showed similar trends in the correlation with the DTI indices, with FA values negatively correlated and $\mathrm{RD}$ values positively correlated.

\section{Discussion}

Our previous study found that anxious depression is associated with poorer outcomes and suggested applying dimensional assessment of co-occurring anxious features of MDD patients, which is feasible for clinical practice in China; then, the clinical classification of anxiety-type depression can achieve better stability and reliability. ${ }^{45}$
So, we used the dimensional assessment of anxious depression in this study to define the anxious subtype of MDD. The HAMD-17 total scores in the anxious group were significantly higher, which is consistent with our previous study ${ }^{45}$ and other studies. ${ }^{6-9}$ Even when excluding the anxiety/somatization factor scores, HAMD total scores were still significantly higher, indicating that the baseline severity of depression in anxious depression is much greater, which means much more challenge in treatment.

Because only the first-episode, medication-free, young anxious or nonanxious MDD patients were included, this is one of the first studies exploring the whole-brain WM neurobiological changes in anxious depression with a TBSS-based

Table 2 Locations of significant $(P<0.05$, FWE corrected) group differences between anxious and nonanxious depressions in FA and RD

\begin{tabular}{|c|c|c|c|c|c|c|c|c|c|c|}
\hline \multirow[t]{2}{*}{$\begin{array}{l}\text { Anxious vs } \\
\text { nonanxious }\end{array}$} & \multirow[t]{2}{*}{$\begin{array}{l}\text { Cluster } \\
\text { index }\end{array}$} & \multirow[t]{2}{*}{$\begin{array}{l}\text { Cluster } \\
\text { voxels }\end{array}$} & \multicolumn{3}{|c|}{$\begin{array}{l}\text { MNI } \\
\text { coordinates } \\
\text { of peak side } \\
\text { voxel }\end{array}$} & \multicolumn{2}{|c|}{$\begin{array}{l}\text { Mean FA or RD } \\
\text { (post hoc) }\end{array}$} & \multirow[t]{2}{*}{ Cohen's d } & \multirow[t]{2}{*}{ Side } & \multirow[t]{2}{*}{ Anatomical regions } \\
\hline & & & $x$ & $y$ & $\mathbf{z}$ & $\begin{array}{l}\text { Anxious } \\
(n=39)\end{array}$ & $\begin{array}{l}\text { Nonanxious } \\
(n=45)\end{array}$ & & & \\
\hline \multirow[t]{3}{*}{ FA } & 3 & 3,457 & -23 & -31 & 34 & 0.58 & 0.65 & 1.35 & $\begin{array}{l}\text { Bilateral } \\
\text { Left }\end{array}$ & $\begin{array}{l}\text { Forceps minor } \\
\text { Superior longitudinal fasciculus, Unicinate } \\
\text { fasciculus }\end{array}$ \\
\hline & 2 & 578 & 31 & -60 & 13 & 0.57 & 0.63 & 1.78 & $\begin{array}{l}\text { Bilateral } \\
\text { Left }\end{array}$ & $\begin{array}{l}\text { Forceps major } \\
\text { Superior longitudinal fasciculus }\end{array}$ \\
\hline & I & 102 & -33 & -51 & 27 & 0.47 & 0.54 & 0.89 & Left & Superior longitudinal fasciculus \\
\hline RD & I & $|2,54|$ & -29 & -3 & 22 & 5.78 & 5.25 & 0.83 & $\begin{array}{l}\text { Bilateral } \\
\text { Left }\end{array}$ & $\begin{array}{l}\text { Forceps major, forceps minor } \\
\text { Superior longitudinal fasciculus }\end{array}$ \\
\hline
\end{tabular}

Notes: Anxious: anxious depression group; nonanxious: nonanxious depression group. The units of RD was $10^{-4} \mathrm{~mm}^{2} / \mathrm{s}$.

Abbreviations: FA, fractional anisotropy; FWE, family-wise error; MNI, Montreal Neurological Institute; RD, radical diffusivity. 

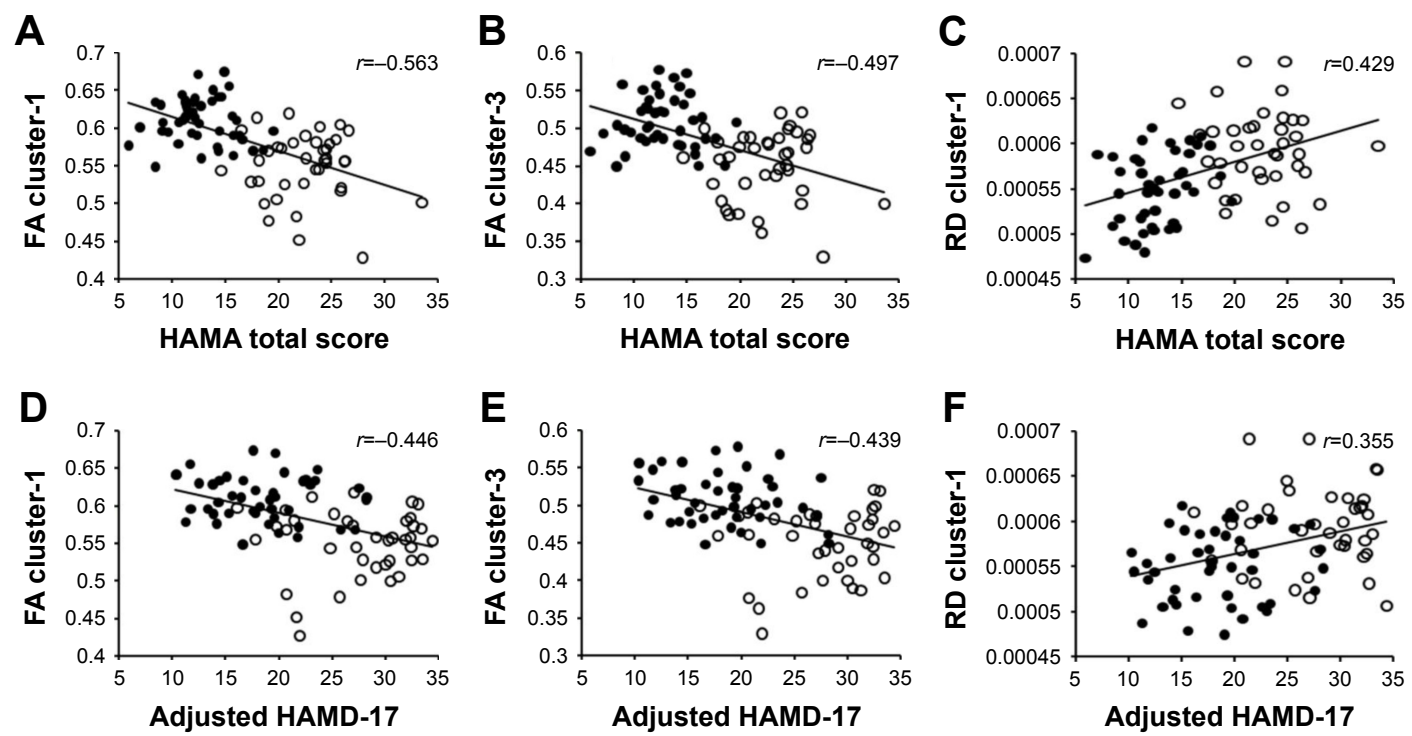

○ Anxious $\bullet$ Nonanxious

Figure 2 Correlation of FA and RD values with clinical features in pooled depression group.

Notes: (A) Mean FA values in cluster-I and HAMA total scores ( $r=-0.563, P<0.00$ I). (B) Mean FA values in cluster-3 and HAMA total scores $(r=-0.497, P<0.00$ I). (C) Mean RD values in cluster-I and HAMA total scores $(r=0.429, P<0.00 I)$. (D) Mean FA values in cluster-I and adjusted HAMD-I7 scores $(r=-0.446, P<0.00 \mathrm{I})$. (E) Mean FA values in cluster-3 and adjusted HAMD- 17 scores $(r=-0.439, P<0.00 I)$. (F) Mean RD values in cluster-I and adjusted HAMD-I7 scores $(r=0.355, P<0.00 \mathrm{I})$.

Abbreviations: FA, fractional anisotropy; HAMA, Hamilton Rating Scale for Anxiety; HAMD, Hamilton Rating Scale for Depression; RD, radial diffusivity.

analysis in such subgroup of patients. Our study could reveal the true nature of anxious subtype MDD and have implications different from other studies which, enrolled mixedaged, medicated or chronic patents.

The main finding of our study are that when compared with the nonanxious subgroup, the FA values of the anxious MDD group had significantly decreased distributed in the projection fibers such as left superior left UF, longitudinal fasciculus (SLF), and bilateral forceps major, the RD values differed significantly in left SLF and forceps major and minor between the two subtype MDD groups, and FA and $\mathrm{RD}$ values of pooled depressive groups were correlated with both anxiety levels and the adjusted depression levels.

The findings supported our hypothesis that the WM lesions mainly located in the fiber pathways related to emotional and cognitive regulation in the anxious depression patients compared to the nonanxious depression subjects, and the effects of higher anxiety level together with higher depression level would lead to more severe deficits in the WM. WM health would be compromised in both anxiety and depression, so, anxious depression could present compounded alternations.

Our results highlighted mainly four WM fiber tracts as follows:

The UF links the limbic structures, connecting the hippocampus and amygdala to the prefrontal regions. It is traditionally considered to be part of the limbic system.
Although the function is not fully known, it might play a role in reward-based learning, negative emotional-cognitive processing bias, declarative memory, and auto noetic selfawareness in psychopathological mechanisms, including that of MDD. ${ }^{51-53} \mathrm{Li}$ et $\mathrm{al}^{54}$ used positron emission tomography scans in investigating the impaired function of prefrontal cortex and found that pronounced hypofrontality, with the associated attentional deficits, plays a key role in the neuropathology of medication resistant depression.

The UF has the longest period of development. It is among the major white fiber tracks that continue to develop beyond the age of 30 years. Eluvathingal et $\mathrm{al}^{55}$ reported that the UF shows lower FA values in socioemotionally deprived children with cognitive, socioemotional, and behavioral difficulties. The age of our subjects is ranging from 20 to 40 years, within the development period of UF, which means that the subjects would be more vulnerable to psychopathological impairment. That maybe one of the explanations of the significant impairment in WM of the patients we enrolled, including the anxious depressive patients.

Abnormalities in the UF fiber bundles associate with social anxiety ${ }^{56}$ and depression that had previously been present in adolescence or early adulthood.$^{57}$ It also associates with cognition functions such as general intelligence, verbal and visual memory, and executive performance. ${ }^{58}$ Studies on the WM of anxiety disorders more consistently 
found decreased FA values in UF, while among the literature of depression studies, some found decreased FA values in UF, and some found no differences. ${ }^{25,31}$ Because our study focused on anxious depression patients, those who suffered from both anxiety and depression, it could be more possible to find the alterations in DTI measures. A possible explanation of the role of UF in depression might be that smaller hippocampus associated with depression might affect the connecting fibers, and uncinated structural alternation that associated with socio-economic deprivation might also play a critical role in the pathogenesis of depression together with the other impaired functions of UF in cognition such as executive performance.

Our study identified significantly decreased FA in the left SLF when compared the anxious group with the nonanxious group, which partially replicated the results of several previous studies. Cole et $\mathrm{al}^{59}$ reported extensive WM impairment in SLF of MDD patients. Dalby et $\mathrm{al}^{60}$ found that lower FA values were associated with more severe depressive symptoms. Murphy and Frodl ${ }^{41}$ ascertained in a meta-analysis that impaired WM integrity of the SLF may be a stable biomarker in the pathophysiology of MDD.

SLF is involved in cognitive and emotional functions. Better sensitivity, sustained ability of attention, and better working memory performance were correlated with increased FA values in the SLF. Some even proposed that FA values of the left SLF combined with a visual evaluation of the anterior thalamic radiation might be helpful in depression diagnosis. ${ }^{61}$ Longer duration of illness, more severity, and longer duration of untreated period might be associated with more WM impairment in SLF of MDD patients. ${ }^{21,29,62}$ Our finding on alternated WM integrity in the SLF might suggest that anxious MDD patients might have more significant impairments in the SLF than in nonanxious patients, which might lead to more impaired cognitive and emotional functions, corresponding to more severe symptoms. The impaired FA of SLF also corresponds to the "cortico-limbic-striato-pallido-thalamic" circuit hypothesis of MDD. ${ }^{63}$

Besides UF and SLF, we found that patients with anxious MDD had lower FA and higher RD in the forceps minor and major of the $\mathrm{CC}$. The $\mathrm{CC}$ is the largest $\mathrm{WM}$, playing a critical role in the communication between the two cerebral hemispheres, integrating high-level cognitions. The forceps minor is constituted by the lateral expansion of fibers of the genu of the $\mathrm{CC}$, which interconnect the dorsolateral prefrontal cortices (DLPFC) ${ }^{64}$ that include portions of the middle and superior frontal gyri. The role of the DLPFC and its connections in the pathogenesis of depression have been described by several previous studies. ${ }^{65,66}$ DLPFC is also known to be involved in attention, executive functions, and internally guided behavior during goal-oriented and working memory tasks. ${ }^{67}$

The forceps major, also known as the posterior forceps, is a fiber bundle, which connects the occipital lobes and crosses the midline via the splenium of the $\mathrm{CC}$. WM alternation in forceps major of MDD was found in many studies. Shen et $\mathrm{al}^{68}$ reported that in forceps major, decreased FA was also found in overall MDD patients compared with healthy controls, but no specific change in FA in recurrent MDD was observed.

We also found increased RD, which is associated with myelin damage, helps to interpret FA changes in patients with anxious depression.

We observed that FA and RD values of pooled depressive groups were correlated with anxiety levels and adjusted depression levels in certain brain clusters, including brain regions such as forceps major, SLF, and UF. Several previous studies also examined the relationship between the neuroimaging parameters and MDD clinical features. Liu et $\mathrm{a}^{40}$ reported that increased fractional amplitude of lowfrequency fluctuation values in the ventral anterior cingulate cortex (ACC) were associated with the severity of anxiety of both anxious depression and remitted depression, proposing that increased resting state activity in the ventral ACC might also be a neuroimaging marker of anxious depression. There are limited available studies on WM microstructure in subtype of depression. Modi et $\mathrm{al}^{69}$ reported a positive relation between trait anxiety levels of subjects and the FA of fornix and left UF, which is partially consistent with our findings. Focusing on anxious depression, Delaparte et $\mathrm{al}^{70}$ used ROI and tractography methods to calculate volumes and FA in the amygdala and UF and found no significant differences compared to nonanxious depression and no significant associations between WM FA and depression severity, this maybe a result of differing diagnostic criteria and different methodology of the imaging analysis, for example, they defined anxious depression in a co-morbid diagnosis, and using a different analysis technique: ROI, which is less sensitive.

No higher FA or lower RD was found in anxious MDD subjects compared with nonanxious MDD ones. The probable explanation is the distinct clinical features of the two groups of patients (significantly different levels of HAMD and HAMA scores, as shown in Table 1). With more severity both in anxiety and depression, anxious MDD might suffer 
more WM impairment, which turned out to be lower FA and higher RD.

Overall, our findings suggest that impairment in WM microstructure in anxious patients is more severe than the nonanxious patients. The impairment could be detected at the early stage of the disorder and is associated with the clinical features.

\section{Limitation}

Further investigation with a larger sample size and longitudinal studies are required to better understand the neuropathology of WM integrity in anxious MDD. Since only young adult subjects were included, we could not directly explore age-related changes in DTI parameters, and a larger age range is required to investigate dynamic changes of WM integrity. To be biological markers of medication treatment, our findings still need to be further validated in future studies.

\section{Conclusion}

From the findings of our study, it can be concluded that significant heterogeneity in alterations in WM micro-integrity, major in projection fibers such as SLF and UF might be helpful to identify the subtype of MDD at the early phase of the disorder, and possible biomarkers for young adult patients with anxious MDD, which is defined by clinical characteristics so far.

\section{Acknowledgments}

This study is supported by National Key Research and Development Program (2016YFC1307100 and 2016YFC1307102), National Natural Science Foundation of China (91232719, 81201056, and 81301159), The National Key Clinical Disciplines at Shanghai Mental Health Center (OMA-MH, 2011-873), and Shanghai Key Laboratory of Psychotic Disorders. The funders played no role in the study design, data collection and analysis, decision to publish, or preparation of the article. The authors thank Xiaoyun Guo, Yousong Su, Jia Huang, Jingjing Xu, Chen Zhang, Zhenghui Yi, Weihong Lu, Zhiguo Wu, Yong Wang, Lan Cao, Yingyan Hu, Chengmei Yuan, and Wu Hong, for their collaboration and help in the study and thank all participants of this study.

\section{Disclosure}

The authors report no conflicts of interest in this work.

\section{References}

1. Rush AJ, Trivedi MH, Wisniewski SR, et al. Acute and longer-term outcomes in depressed outpatients requiring one or several treatment steps: a STAR*D report. Am J Psychiatry. 2006;163(11):1905-1917.
2. Uher R, Muthen B, Souery D, et al. Trajectories of change in depression severity during treatment with antidepressants. Psychol Med. 2010; 40(8):1367-1377.

3. Kroenke K, Spitzer RL, Williams JB, Monahan PO, Lowe B. Anxiety disorders in primary care: prevalence, impairment, comorbidity, and detection. Ann Intern Med. 2007;146(5):317-325.

4. Pini S, Cassano GB, Simonini E, Savino M, Russo A, Montgomery SA. Prevalence of anxiety disorders comorbidity in bipolar depression, unipolar depression and dysthymia. J Affect Disord. 1997;42(2-3): 145-153.

5. Fava M, Rush AJ, Alpert JE, et al. What clinical and symptom features and comorbid disorders characterize outpatients with anxious major depressive disorder: a replication and extension. Can J Psychiatry. 2006;51(13):823-835.

6. Fava M, Martinez JM, Greist J, et al. The efficacy and tolerability of duloxetine in the treatment of anxious versus non-anxious depression: a post-hoc analysis of an open-label outpatient study. Ann Clin Psychiatry. 2007;19(3):187-195.

7. Joffe RT, Bagby RM, Levitt A. Anxious and nonanxious depression. Am J Psychiatry. 1993;150(8):1257-1258.

8. Tollefson GD, Holman SL, Sayler ME, Potvin JH. Fluoxetine, placebo, and tricyclic antidepressants in major depression with and without anxious features. J Clin Psychiatry. 1994;55(2):50-59.

9. Clayton PJ, Grove WM, Coryell W, Keller M, Hirschfeld R, Fawcett J. Follow-up and family study of anxious depression. Am J Psychiatry. 1991;148(11):1512-1517.

10. Savitz JB, Drevets WC. Imaging phenotypes of major depressive disorder: genetic correlates. Neuroscience. 2009;164(1):300-330.

11. Liao $\mathrm{Y}$, Huang $\mathrm{X}, \mathrm{Wu} \mathrm{Q}$, et al. Is depression a disconnection syndrome? Meta-analysis of diffusion tensor imaging studies in patients with MDD. J Psychiatry Neurosci. 2013;38(1):49-56.

12. Kubicki M, Westin CF, Maier SE, et al. Diffusion tensor imaging and its application to neuropsychiatric disorders. Harv Rev Psychiatry. 2002;10(6):324-336.

13. Korgaonkar MS, Williams LM, Song YJ, Usherwood T, Grieve SM. Diffusion tensor imaging predictors of treatment outcomes in major depressive disorder. Br J Psychiatry. 2014;205(4):321-328.

14. Ahn S, Lee SK. Diffusion tensor imaging: exploring the motor networks and clinical applications. Korean J Radiol. 2011;12(6):651-661.

15. Le Bihan D, Mangin JF, Poupon C, et al. Diffusion tensor imaging: concepts and applications. J Magn Reson Imaging. 2001;13(4): 534-546.

16. Taylor WD, Hsu E, Krishnan KR, MacFall JR. Diffusion tensor imaging: background, potential, and utility in psychiatric research. Biol Psychiatry. 2004;55(3):201-207.

17. Basser PJ. Inferring microstructural features and the physiological state of tissues from diffusion-weighted images. NMR Biomed. 1995; 8(7-8):333-344.

18. Song SK, Yoshino J, Le TQ, et al. Demyelination increases radial diffusivity in corpus callosum of mouse brain. Neuroimage. 2005;26(1): $132-140$.

19. Smith SM, Jenkinson M, Johansen-Berg H, et al. Tract-based spatial statistics: voxelwise analysis of multi-subject diffusion data. Neuroimage. 2006;31(4):1487-1505.

20. Ouyang $\mathrm{X}$, Tao HJ, Liu HH, et al. White matter integrity deficit in treatment-naive adult patients with major depressive disorder. East Asian Arch Psychiatry. 2011;21(1):5-9.

21. Jia Z, Huang X, Wu Q, et al. High-field magnetic resonance imaging of suicidality in patients with major depressive disorder. Am J Psychiatry. 2010;167(11):1381-1390.

22. Ma N, Li L, Shu N, et al. White matter abnormalities in first-episode, treatment-naive young adults with major depressive disorder. $\mathrm{Am} \mathrm{J}$ Psychiatry. 2007;164(5):823-826.

23. Tha KK, Terae S, Nakagawa $\mathrm{S}$, et al. Impaired integrity of the brain parenchyma in non-geriatric patients with major depressive disorder revealed by diffusion tensor imaging. Psychiatry Res. 2013;212(3): 208-215. 
24. Wang T, Huang X, Huang P, et al. Early-stage psychotherapy produces elevated frontal white matter integrity in adult major depressive disorder. PLoS One. 2013;8(4):e63081.

25. Cullen KR, Klimes-Dougan B, Muetzel R, et al. Altered white matter microstructure in adolescents with major depression: a preliminary study. J Am Acad Child Adolesc Psychiatry. 2010;49(2):173.e1-183.e1.

26. Han KM, Choi S, Jung J, et al. Cortical thickness, cortical and subcortical volume, and white matter integrity in patients with their first episode of major depression. J Affect Disord. 2014;155:42-48.

27. Olvet DM, Peruzzo D, Thapa-Chhetry B, et al. A diffusion tensor imaging study of suicide attempters. J Psychiatr Res. 2014;51:60-67.

28. Lai $\mathrm{CH}, \mathrm{Wu} \mathrm{YT}$. Alterations in white matter micro-integrity of the superior longitudinal fasciculus and anterior thalamic radiation of young adult patients with depression. Psychol Med. 2014;44(13): $2825-2832$.

29. Wu F, Tang Y, Xu K, et al. Whiter matter abnormalities in medicationnaive subjects with a single short-duration episode of major depressive disorder. Psychiatry Res. 2011;191(1):80-83.

30. Zuo N, Fang J, Lv X, et al. White matter abnormalities in major depression: a tract-based spatial statistics and rumination study. PLoS One 2012;7(5):e37561.

31. Olvet DM, Delaparte L, Yeh FC, et al. A comprehensive examination of white matter tracts and connectometry in major depressive disorder Depress Anxiety. 2016;33(1):56-65.

32. Andreescu C, Wu M, Butters MA, Figurski J, Reynolds CF 3rd, Aizenstein HJ. The default mode network in late-life anxious depression. Am J Geriatr Psychiatry. 2011;19(11):980-983.

33. Alexopoulos GS, Kiosses DN, Choi SJ, Murphy CF, Lim KO. Frontal white matter microstructure and treatment response of late-life depression a preliminary study. Am J Psychiatry. 2002;159(11):1929-1932.

34. Kessler D, Sharp D, Lewis G. Screening for depression in primary care. Br J Gen Pract. 2005;55(518):659-660.

35. Yuan $\mathrm{Y}$, Zhang Z, Bai F, et al. White matter integrity of the whole brain is disrupted in first-episode remitted geriatric depression. Neuroreport 2007;18(17):1845-1849.

36. Nobuhara K, Okugawa G, Sugimoto T, et al. Frontal white matter anisotropy and symptom severity of late-life depression: a magnetic resonance diffusion tensor imaging study. J Neurol Neurosurg Psychiatry. 2006;77(1):120-122.

37. Brodaty H, Cullen B, Thompson C, et al. Age and gender in the phenomenology of depression. Am J Geriatr Psychiatry. 2005;13(7):589-596.

38. Giorgio A, Watkins KE, Chadwick M, et al. Longitudinal changes in grey and white matter during adolescence. Neuroimage. 2010;49(1): 94-103.

39. Lijster JM, Dierckx B, Utens EM, et al. The age of onset of anxiety disorders. Can J Psychiatry. 2017;62(4):237-246.

40. Liu CH, Ma X, Song LP, et al. Abnormal spontaneous neural activity in the anterior insular and anterior cingulate cortices in anxious depression. Behav Brain Res. 2015;281:339-347.

41. Murphy ML, Frodl T. Meta-analysis of diffusion tensor imaging studies shows altered fractional anisotropy occurring in distinct brain areas in association with depression. Biol Mood Anxiety Disord. 2011; 1(1):3.

42. Wise T, Radua J, Nortje G, Cleare AJ, Young AH, Arnone D. Voxel-based meta-analytical evidence of structural disconnectivity in major depression and bipolar disorder. Biol Psychiatry. 2016;79(4):293-302.

43. Chakos MH, Schobel SA, Gu H, et al. Duration of illness and treatment effects on hippocampal volume in male patients with schizophrenia. Br J Psychiatry. 2005;186:26-31.

44. de Diego-Adelino J, Pires P, Gomez-Anson B, et al. Microstructural white-matter abnormalities associated with treatment resistance, severity and duration of illness in major depression. Psychol Med. 2014; 44(6):1171-1182.

45. Wu Z, Chen J, Yuan C, et al. Difference in remission in a Chinese population with anxious versus nonanxious treatment-resistant depression: a report of OPERATION study. J Affect Disord. 2013;150(3): 834-839.
46. Fava M, Alpert JE, Carmin CN, et al. Clinical correlates and symptom patterns of anxious depression among patients with major depressive disorder in STAR*D. Psychol Med. 2004;34(7):1299-1308.

47. Fava M, Rush AJ, Alpert JE, et al. Difference in treatment outcome in outpatients with anxious versus nonanxious depression: a STAR*D report. Am J Psychiatry. 2008;165(3):342-351.

48. Hamilton M. A rating scale for depression. J Neurol Neurosurg Psychiatry. 1960;23:56-62.

49. Johansen-Berg H, Behrens TE, Robson MD, et al. Changes in connectivity profiles define functionally distinct regions in human medial frontal cortex. Proc Natl Acad Sci U S A. 2004;101(36):13335-13340.

50. Vaccarino AL, Evans KR, Sills TL, Kalali AH. Symptoms of anxiety in depression: assessment of item performance of the Hamilton Anxiety Rating Scale in patients with depression. Depress Anxiety. 2008;25(12): 1006-1013.

51. Gottfried JA, O'Doherty J, Dolan RJ. Encoding predictive reward value in human amygdala and orbitofrontal cortex. Science. 2003; 301(5636):1104-1107.

52. Mabbott DJ, Rovet J, Noseworthy MD, Smith ML, Rockel C. The relations between white matter and declarative memory in older children and adolescents. Brain Res. 2009;1294:80-90.

53. Rodrigo S, Naggara O, Oppenheim C, et al. Human subinsular asymmetry studied by diffusion tensor imaging and fiber tracking. AJNR Am J Neuroradiol. 2007;28(8):1526-1531.

54. Li CT, Su TP, Wang SJ, Tu PC, Hsieh JC. Prefrontal glucose metabolism in medication-resistant major depression. Br J Psychiatry. 2015; 206(4):316-323

55. Eluvathingal TJ, Chugani HT, Behen ME, et al. Abnormal brain connectivity in children after early severe socioemotional deprivation: a diffusion tensor imaging study. Pediatrics. 2006;117(6):2093-2100.

56. Phan KL, Orlichenko A, Boyd E, et al. Preliminary evidence of white matter abnormality in the uncinate fasciculus in generalized social anxiety disorder. Biol Psychiatry. 2009;66(7):691-694.

57. Taylor WD, MacFall JR, Gerig G, Krishnan RR. Structural integrity of the uncinate fasciculus in geriatric depression: relationship with age of onset. Neuropsychiatr Dis Treat. 2007;3(5):669-674.

58. Nakamura M, McCarley RW, Kubicki M, et al. Fronto-temporal disconnectivity in schizotypal personality disorder: a diffusion tensor imaging study. Biol Psychiatry. 2005;58(6):468-478.

59. Cole J, Chaddock CA, Farmer AE, et al. White matter abnormalities and illness severity in major depressive disorder. Br J Psychiatry. 2012; 201(1):33-39.

60. Dalby RB, Frandsen J, Chakravarty MM, et al. Depression severity is correlated to the integrity of white matter fiber tracts in late-onset major depression. Psychiatry Res. 2010;184(1):38-48.

61. Niida A, Niida R, Kuniyoshi K, Motomura M, Uechi A. Usefulness of visual evaluation of the anterior thalamic radiation by diffusion tensor tractography for differentiating between Alzheimer's disease and elderly major depressive disorder patients. Int J Gen Med. 2013;6:189-200.

62. Kieseppa T, Eerola M, Mantyla R, et al. Major depressive disorder and white matter abnormalities: a diffusion tensor imaging study with tractbased spatial statistics. J Affect Disord. 2010;120(1-3):240-244.

63. Sheline YI. 3D MRI studies of neuroanatomic changes in unipolar major depression: the role of stress and medical comorbidity. Biol Psychiatry. 2000;48(8):791-800.

64. Voineskos AN, Farzan F, Barr MS, et al. The role of the corpus callosum in transcranial magnetic stimulation induced interhemispheric signal propagation. Biol Psychiatry. 2010;68(9):825-831.

65. Koenigs M, Grafman J. The functional neuroanatomy of depression: distinct roles for ventromedial and dorsolateral prefrontal cortex. Behav Brain Res. 2009;201(2):239-243.

66. Sexton CE, Mackay CE, Ebmeier KP. A systematic review of diffusion tensor imaging studies in affective disorders. Biol Psychiatry. 2009; 66(9):814-823.

67. Demeter E, Hernandez-Garcia L, Sarter M, Lustig C. Challenges to attention: a continuous arterial spin labeling (ASL) study of the effects of distraction on sustained attention. Neuroimage. 2011;54(2):1518-1529. 
68. Shen X, Reus LM, Cox SR, et al. Subcortical volume and white matter integrity abnormalities in major depressive disorder: findings from UK Biobank imaging data. Sci Rep. 2017;7(1):5547.

69. Modi S, Trivedi R, Singh K, et al. Individual differences in trait anxiety are associated with white matter tract integrity in fornix and uncinate fasciculus: preliminary evidence from a DTI based tractography study. Behav Brain Res. 2013;238:188-192.
70. Delaparte L, Yeh FC, Adams P, et al. A comparison of structural connectivity in anxious depression versus non-anxious depression. J Psychiatr Res. 2017;89:38-47.

\section{Publish your work in this journal}

Neuropsychiatric Disease and Treatment is an international, peerreviewed journal of clinical therapeutics and pharmacology focusing on concise rapid reporting of clinical or pre-clinical studies on a range of neuropsychiatric and neurological disorders. This journal is indexed on PubMed Central, the 'PsycINFO' database and CAS, and is the official journal of The International Neuropsychiatric Association (INA). The manuscript management system is completely online and includes a very quick and fair peer-review system, which is all easy to use. Visit http://www.dovepress.com/testimonials.php to read real quotes from published authors.

Submit your manuscript here: http://www.dovepress.com/neuropsychiatric-disease-and-treatment-journal 